\title{
Sexualidade na terceira idade e educação em saúde: um relato de experiência
}

\author{
Sexuality in old age and health education: an \\ experience report
}

Isadora Carolina Calaça de Lima ${ }^{1}$, Sarah Larissa Reis Fernandes ${ }^{2}$,Guilherme Roberto Naves Miranda $^{3}$, Heloísa Silva Guerra ${ }^{4}$, Rayana Gomes Oliveira Loreto ${ }^{5}$

1. Universidade de Rio Verde (UniRV) - Campus Aparecida de Goiânia - Brasil. E-mail: i_sadora@hotmail. com ORCID: https://orcid.org/0000-0002-4665-606X

2. Universidade de Rio Verde (UniRv) - Campus Aparecida de Goiânia - Brasil. E-mail: sarah_larissa_ fernandes@hotmail.com ORCID: https://orcid.org/0000-0002-3796-2073

3. Universidade para o Desenvolvimento do Estado e da Região do Pantanal (Uniderp) - Brasil. E-mail: guilhermernm9704@hotmail.com ORCID: https://orcid.org/0000-0001-7425-0262

4. Universidade de Rio Verde (UniRV) - Campus Aparecida de Goiânia, Brasil. E-mail: heloisaguerra@unirv. edu.br ORCID: https://orcid.org/0000-0001-6429-5424

5. Pontifícia Universidade Católica de Goiás (PUC-GO) - Brasil. E-mail: rayana.loreto@hotmail.com ORCID: https://orcid.org/0000-0002-9372-6745

CONTATO: Isadora Carolina Calaça de Lima | Alameda Pedro de Sá, s/n - Quadra CHC - Lote 21E, C - Jardim dos Buritis | Aparecida de Goiânia | Goiás | E-mail: i_sadora@hotmail.com

COMO CITAR: Lima ICC, Fernandes SLR, Miranda GRN, Guerra HS, Loreto RGO. Sexualidade na terceira idade e educação em saúde: um relato de experiência. R. Saúde Públ. Paraná. 2020 Jul;3(1):137-143.

RESUMO A pirâmide etária mostra um envelhecimento progressivo no mundo. Esse processo envolve aspectos relacionados à saúde, entre eles a sexualidade como variável interferente na qualidade de vida do ser humano. Este relato buscou discorrer sobre uma ação de educação em saúde voltada para os idosos, realizada no âmbito da 
Atenção Primária em Saúde, desenvolvida por estudantes de medicina. A experiência ocorreu por meio de rodas de conversa e aconselhamento acerca do tema sexualidade na terceira idade, abordando assuntos referentes aos preconceitos e tabus nessa fase, além das mudanças decorrentes do envelhecimento e alertas sobre as Infecções Sexualmente Transmissíveis (ISTs). Dessa forma, a dinâmica estabelecida teve como foco principal contribuir para o empoderamento dos idosos, tornando-os capazes de discernir os mitos e estereótipos da sexualidade, bem como instruir quanto aos riscos e as formas de prevenção às ISTs.

PALAVRAS-CHAVE: Sexualidade. Envelhecimento. Doenças Sexualmente Transmissíveis. Promoção da Saúde. Saúde do Idoso.

\begin{abstract}
The age pyramid shows progressive ageing in the world. This process involves aspects related to health, including sexuality as an interfering variable in the quality of life of the human being. This report aimed to discuss a health education action directed to senior citizens, carried out within the scope of Primary Health Care, developed by medical students. The experience took place through conversation and counseling concerning the theme 'sexuality in old age', addressing issues related to prejudice and taboos in this phase, as well as changes due to aging and warnings about Sexually Transmitted Infections (STIs). Thus, the established dynamics had as main focus to contribute to the empowerment of the elderly, making them capable of discerning the myths and stereotypes of sexuality, as well as instructing on the risks and ways to prevent STIs.
\end{abstract}

KEYWORDS: Sexuality. Aging. Sexually Transmitted Diseases. Health Promotion. Health of the Elderly.

\title{
INTRODUÇÃo
}

A pirâmide etária revela um envelhecimento progressivo da população mundial. Estima-se que, em 2050, uma em cada cinco pessoas no mundo terá mais de 60 anos, representando dois bilhões de pessoas'. As transformações no padrão demográfico iniciaram-se a partir da década de 40, com o declínio da taxa de mortalidade, e acentuou-se após 1960 em decorrência de quedas expressivas dos níveis de fecundidade 2 . Paralelamente a este fenômeno, houve uma elevação da expectativa de vida da população, atribuída principalmente aos avanços tecnológicos da ciência, novos medicamentos, com consequente cura de diversas doenças, interferindo no processo de envelhecimento ${ }^{3}$.

O envelhecimento traduz-se como um processo biopsicossocial constituído por mudanças físicas, psicológicas, fisiológicas e nos papéis sociais ${ }^{3}$. 0 efeito desse processo relacionado à sexualidade é dotado de estereótipos e preconceitos, o que torna o assunto negligenciado. No entanto, a função sexual continua 
até mesmo na terceira idade ${ }^{4}$. Além disso, há uma escassez de informações quanto à sexualidade na terceira idade, o que contribui para a manutenção dos tabus em nossa sociedade.

No Brasil, a Política Nacional do Idoso considera como idoso a pessoa maior de sessenta anos de idade ${ }^{5}$. Durante a velhice ocorrem modificações físicas e fisiológicas como aparecimento de rugas, cabelos brancos, diminuição da elasticidade da pele, encurtamento postural, problemas de circulação, desaceleração do metabolismo, pele mais seca, limitação do campo visual, diminuição da massa muscular, entre outras. Ademais, na mulher ocorre diminuição dos hormônios como o estrógeno e a progesterona, reduzindo a lubrificação vaginal e interferindo na perda do desejo sexual. No homem há uma progressiva redução da espermatogênese e retardo na ereção durante a fase de excitação e redução da congestão vascular, contudo o interesse sexual é mantido ${ }^{6.7}$.

A ideia de que a velhice é um período de decadências juntamente com a pressão social exercida sobre os idosos, torna a sexualidade reprimida, o que acaba por inibir a vida sexual de grande parte dessa classe etária. Com isso, muitas vezes os idosos deixam suas vontades e desejos de lado por medo e sentimento de culpa para com a sociedade, que ainda hoje manifesta um grande preconceito em relação a essa fase da vida ${ }^{8}$.

O sexo, quando analisado sob a perspectiva da procriação, torna-se menos necessário com a idade. As alterações hormonais, especialmente na mulher após a menopausa, resultam em uma redução do desejo sexual durante a velhice. Entretanto, a sexualidade relacionada ao envelhecimento remete a mitos e estereótipos, levando idosos a parecerem assexuados e, consequentemente, representando um tabu ${ }^{9}$.

O tratamento de reposição hormonal e o uso de Sildenafil ${ }^{\circledR}$ proporcionaram aos idosos a continuidade da busca pelo prazer sexual. No entanto, as práticas sexuais inseguras e o conhecimento limitado quanto às infecções sexualmente transmissíveis (ISTs) tornam essa população mais vulnerável às doenças, visto que em épocas anteriores não se tinha campanhas de educação em saúde e orientações quanto à necessidade de prevenção $0^{10.11}$. Segundo o Ministério da Saúde, em 2018, entre as mulheres com 60 anos ou mais, houve um aumento de $21,2 \%$ comparativamente aos últimos dez anos na detecção de AIDS, e entre os homens a taxa de detecção foi de 13,4 para cada 100 mil habitantes, refletindo a alta incidência dos casos dessa IST'2.

A abordagem da temática da sexualidade é complexa, uma vez que envolve vários aspectos da vida do ser humano, como o social, cultural, psicológico e físico. Dessa maneira, faz-se necessária uma capacitação profissional no âmbito da Atenção Primária, para que se possa abordar com eficiência esse tema nas consultas com os idosos, a fim de prevenir doenças e promover a saúde coletiva e individual. As Unidades Básicas de Saúde (UBSs) são a porta de entrada para o atendimento da população e o principal vínculo da sociedade com o setor de saúde. Logo, cria-se uma relação de confiança, alcançando maior sucesso na terapêutica ${ }^{13}$.

A educação em saúde é imprescindivel nesse processo, buscando promover o envelhecimento ativo e otimizar as capacidades dos idosos, contribuindo para sua qualidade de vida. Nessa perspectiva, os profissionais de saúde devem estar preparados durante o atendimento para que os idosos se sintam à vontade para conversar, explicitando as orientações em prol da promoção da saúde ${ }^{8.14}$. em todos os níveis de atenção. Este estudo teve como objetivo relatar a experiência de uma atividade de educação em saúde voltada para a terceira idade priorizando a temática sexualidade.

\section{MÉTODO}

Trata-se de um estudo descritivo, do tipo relato de experiência, a partir da vivência de discentes e 
docentes da disciplina "Medicina Integrada à Saúde da Comunidade". A referida disciplina trabalha conteúdos relacionados ao Sistema Único de Saúde e permite aos acadêmicos a vivência em cenários reais de atuação em Saúde Coletiva, com enfoque na Atenção Primária à Saúde. Como metodologia base, a disciplina se utiliza do Arco de Maguerez, que permite por meio de um processo de análise da realidade, que o aluno caminhe de uma visão sincrética, geral e precária para uma visão sintética e mais elaborada sobre a prática ${ }^{15}$.

A Metodologia da Problematização com o Arco de Maguerez tem como ponto de partida e chegada a realidade que, observada sob diversos ângulos, permite ao estudante ou pesquisador extrair e identificar os problemas existentes e propor estratégias para superá-los, transformando assim essa realidade em algum grau.

No desenvolvimento da disciplina, discentes e docentes realizaram um planejamento, além da articulação com a Unidade Básica de Saúde que funciona como campo de atuação para os acadêmicos, onde foram coletadas, por meio de dados secundários da própria UBS, as informações acerca do perfil da população atendida.

A partir das informações da população adscrita, os acadêmicos idealizaram uma proposta educativa, tendo o idoso como público-alvo e a sexualidade como tema central. 0 apoio da equipe de saúde da família tornou possível a viabilização da atividade, concretizada mediante o convite aos idosos da comunidade e oferta do espaço para o desenvolvimento da proposta.

A atividade foi realizada na própria UBS pelos acadêmicos do $6^{\circ}$ período do curso de Medicina de uma fundação pública de ensino do interior de Goiás. Contou com 30 participantes, sendo 21 mulheres e 9 homens, além dos 22 discentes envolvidos e da equipe da unidade.

Na dinâmica abordou-se o idoso em sua integralidade, focando nos temas sexualidade na terceira idade, uso racional de medicamentos, vacinação e cuidados na velhice, priorizando temas relacionados à caderneta do idoso.

A ação seguiu os seguintes passos: primeiramente os idosos foram acolhidos e triados por uma equipe de alunos que aferiam a pressão arterial e verificavam a caderneta do Idoso, procurando lacunas no preenchimento, bem como orientar sobre medicamentos, vacinação, manutenção do peso e hábitos saudáveis de vida. Após esse primeiro momento, os idosos eram encaminhados a salas reservadas para discussão dos temas.

Por meio de rodas de conversa o tema da sexualidade na terceira idade foi abordado em salas separadas, contendo no máximo 6 participantes, que foram separados conforme o sexo para possibilitar maior interação durante a discussão e evitar constrangimentos. Além dos idosos, alguns acadêmicos ficaram na sala e apresentaram um banner explicativo com perguntas e respostas frequentes sobre o assunto. Assim, a conversa foi introduzida através de perguntas direcionadas aos participantes, possibilitando uma troca de experiências e discussão sobre o tema que era finalizado com a resposta correta para a pergunta.

Além disso, o banner cujo título era "Vamos falar de Sexo?" continha os assuntos referentes ao preconceito e tabus que os idosos enfrentam no tocante à sexualidade, explicitando que os idosos podem ter relação sexual, bem como as principais mudanças que ocorrem no corpo com o envelhecimento e formas de melhorar o desempenho sexual. Além desses assuntos, o banner também continha tópicos que alertavam sobre as ISTs, sobre a importância do uso de preservativo durante as relações sexuais em qualquer fase da vida e instruções quanto ao uso correto. Por fim, foram distribuídas camisinhas masculinas, femininas e lubrificantes fornecidos pela Unidade de Saúde. 


\section{RESULTADOS E DISCUSSÃO}

Ao final da atividade, de acordo com o relato dos próprios participantes, percebeu-se que os idosos consideraram as informações repassadas quanto à vida sexual, novas e relevantes. Além disso, a grande maioria não tinha conhecimento a respeito da importância do uso de preservativos e quanto à alta prevalência de ISTs nessa fase da vida, em especial as mais prevalentes como sífilis, gonorreia e HIV. Observou-se também. por meio dessa troca, que muitos deles tinham medo de se envolverem sexualmente com seus parceiros por receio de julgamentos externos e coerção social e familiar por se considerarem impotentes pela idade.

A população mundial está envelhecendo, e os avanços tecnológicos e pesquisas na área da saúde possibilitam o tratamento de doenças crônicas e aumentam a expectativa e qualidade de vida das pessoas. Sendo assim, faz-se necessário novos e direcionados investimentos para atender a essa população idosa e proporcionar a dignidade de viver bem ${ }^{14}$.

O homem e a mulher podem continuar a ter relações sexuais durante a terceira idade e devem ser esclarecidos quanto às alterações que ocorrem em ambos para que não haja prejuízos no prazer sexual e nas relações afetivas, adaptando-se às mudanças ocorridas nesta fase ${ }^{16}$.

O desconhecimento dos idosos referente ao crescimento na incidência da AIDS em pessoas mais velhas contribui para um aumento da epidemia, tornando-se uma ameaça à saúde pública. Além disso, o não uso de camisinha constitui um fator de risco importante, pois a maioria dos idosos não se considera como um grupo de risco para contrair a doença ${ }^{16}$.

Para que os idosos vivam uma sexualidade satisfatória, é imprescindível receber uma assistência acolhedora dos profissionais de saúde, por meio de informações dos acontecimentos próprios da velhice e. assim, eliminar suas inseguranças e responder as dúvidas ${ }^{17}$.

A educação em saúde configura-se como uma ferramenta de intervenção pedagógica bem-sucedida por permitir a construção de saberes, o aprimoramento do conhecimento, auxiliando na criação de reflexão para o esclarecimento de dúvidas ${ }^{18}$. A sua realização é feita a partir do diálogo, de forma participativa e da construção de uma relação acolhedora para crescimento e melhora na vida do indivíduo ${ }^{18}$. No âmbito da terceira idade constitui uma ferramenta para aprimorar a qualidade de vida dos idosos e promover o autocuidado 20 .

A longo prazo, os benefícios da educação em saúde se traduzem na diminuição do custo com internações, fármacos para doenças crônicas e tratamentos especializados ${ }^{20}$. A criação da Política Nacional de Saúde da Pessoa Idosa e do Estatuto do Idoso assegurou o direcionamento das ações sociais e de saúde que garantem os direitos das pessoas idosas e obrigam o Estado a proteger esta população ${ }^{20}$.

Desse modo, é preciso alterar o meio de atendimento a essa população, criando uma visão de cuidado em todas as esferas de convívio do indivíduo, uma vez que é muito comum o abandono e negligência com os idosos, o que favorece ainda mais o adoecimento biológico e psicológico dessa população ${ }^{21}$.

Embora fatores fisiológicos e psicossociais tenham impacto sobre a expressão, a sexualidade permanece essencial à qualidade de vida de muitos adultos mais velhos. Os profissionais de saúde devem estar preparados para lidar com componentes tais como a expressão sexual, a disfunção sexual, a identidade e estigma, o comprometimento cognitivo e a capacidade de consentimento e, às vezes, comportamentos sexuais inapropriados visando melhorar a qualidade de vida de idosos ${ }^{22}$. Também devem estar cientes das crescentes necessidades de educação sexual em idosos e serem incentivados a discutir abertamente questões e preocupações sexuais desses pacientes ${ }^{23}$. 
A figura do profissional de saúde torna-se de suma importância no desenvolvimento de atividades educativas para promoção de saúde da população, valorizando sua autonomia, por se tratar de um processo transformador ${ }^{18.19}$. Sendo assim, a educação em saúde sexual na escola de medicina ajuda os alunos a tomarem consciência ou corrigir suas próprias crenças e presunções estereotipadas sobre sexualidade, uma vez que, se estas forem negativas, podem levar a efeitos prejudiciais para pacientes em serviços de saúde ${ }^{24}$.

A elaboração das Diretrizes Curriculares Nacionais (DCN) para os cursos universitários, provocou mudanças na formação, respaldada em competências, habilidades e atitudes para atuarem no Sistema Único de Saúde (SUS). A mudança diz respeito à substituição de um modelo flexneriano, biomédico e curativo para outro, orientado no processo saúde-doença em diferentes níveis de atenção, contemplando ações de promoção, prevenção, recuperação e reabilitação em uma dimensão coletiva ${ }^{19}$. Assim, é imprescindivel a inserção precoce do acadêmico no SUS para desenvolvimento de uma visão holística e crítica, considerando o conceito ampliado de saúde ${ }^{19}$.

\section{CONSIDERAÇÕES FINAIS}

Abordar o tema da sexualidade na terceira idade deve tornar-se rotina nos setores de saúde, tanto públicos como privados. 0 tema tem sido negligenciado atualmente, devido ao reflexo do despreparo dos profissionais de saúde e temor em abordar esse assunto nas consultas. Ter em mente que o envelhecer é algo natural e que o desejo sexual faz parte da vida de todos durante todo o curso da vida, pode auxiliar no atendimento a essa faixa etária, proporcionando confiança e encorajando os pacientes a não abandonarem suas vidas sexuais por influências externas e pressão social.

O presente trabalho atuou nesse aspecto, uma vez que os acadêmicos se propuseram a suscitar dúvidas, gerar curiosidade e interesse pelo assunto nos idosos, a fim de que pudessem continuar vivenciando a sexualidade e buscassem auxílio em casos de mais dúvidas. Logo, conseguiram fazer uma correlação da teoria do atendimento humanizado, preconizado pelo SUS, com a prática na UBS a partir de uma visão holística para que todos tenham o contexto da saúde como um estado de completo bem-estar físico, mental e social, a fim de que a promoção do conhecimento seja um instrumento valioso para o empoderamento da população.

\section{REFERÊNCIAS}

1. Ministério da Saúde (BRASIL). Envelhecimento e Saúde da Pessoa Idosa. Brasília: Ministério da Saúde. 2006.

2. Simões CCS. Relações entre as alterações históricas na dinâmica demográfica brasileira e os impactos decorrentes do processo de envelhecimento da população. Rio de Janeiro: IBGE. 2016.

3. Oliveira LB, Baía RV, Delgado ART, Vieira KFL, Lucena ALR. Sexualidade e envelhecimento: avaliação do perfil sexual de idosos não institucionalizados. Rev Ciênc Saúde Nova Esperança. 2015 Dez:13(2):42-50

4. Almeida T, Lourenço ML. Envelhecimento, amor e sexualidade: utopia ou realidade? Rev Bras Geriatr Gerontol. 2007:10(1):101-114

5. BRASIL. Lei $n^{\circ}$ 8.842, de 04 de janeiro de 1994. Dispõe sobre a política nacional do idoso, cria o Conselho Nacional do Idoso e dá outras providências. Diário Oficial [da] República Federativa do Brasil, Brasília, DF, 05 jan. 1994. Disponivel em: <http://www. planalto.gov.br/ ccivil_03/leis/l8842.htm>. Acesso em: 2 jan. 2019.

6. Biasus F, Demantova A, Camargo BV. Representações sociais do envelhecimento e da sexualidade para pessoas com mais de 50 anos. Temas em Psicol. 2011:19(1):319- 336. 
7. Freitas EV, Py L. Tratado de Geriatria e Gerontologia. 4. ed. Rio de Janeiro: Guanabara Koogan Ltda. 2016.

8. Vieira KFL, Coutinho MPL, Saraiva ERA. A sexualidade na velhice: representações sociais de idosos frequentadores de um grupo de convivência. Psicol Cênc Prof. 2016; 36(1):196-209

9. Santos MC, et al. Percepções e vivências de idosos sobre sexualidade. Almanaque multidisciplinar de pesquisa. 2017:1(1):25,36.

10. Uchôa YS, Costa DCA, Junior IAPS, Silva STSE, Freitas WMTM, Soares SCS. A sexualidade sob o olhar da pessoa idosa. Rev Bras Geriatr Gerontol. 2016;19(6):939-949

11. Neto JD, Nakamura AS, Cortez LER, Yamaguchi UM. Doenças sexualmente transmissiveis em idosos: uma revisão sistemática. Ciênc Saúde Coletiva. 2015:20(12):3853-3864

12. Brasil. Ministério da Saúde (MS). Secretaria de Vigilância em Saúde. Departamento de DST, AIDS e Hepatites Virais. Boletim Epidemiológico AIDS-DST 2018 -2018. [acessado 2019 jan 10]. Disponivel em: http:// http://www.aids.gov.br/pt-br/pub/2018/boletimepidemiologico-hivaids-2018

13. Silva EM, Melo GL, Carvalho MM, Silva JC, Luz VLES. O significado da sexualidade para o idoso assistido pela estratégia saúde da família. Rev Interd. 2011;4(4):30-35

14. Mallmann DG, Neto NMG, Sousa JC, Vasconcelos EMR. Educação em saúde como principal alternativa para promover a saúde do idoso. Ciênc Saúde Coletiva. 2015:20(6):1763-1722

15. Berbel NAN. A metodologia da problematização no ensino superior e sua contribuição para o plano da práxis. Rev Semina. 1996:17:7-12.

16. Jesus DS, Fernandes FP, Coelho ACL, Simões NL, Campos PRC, Ribeiro VC, et al. Nível de conhecimento sobre DST's e a influência da sexualidade na vida integral da mulher idosa. Rev em Foco. 2016;1(5): 33-45.

17. Evangelista AR, Moreira ACA, Freitas CASL, Val DR, Diniz JL, Azevedo SGV. Sexuality in old age: knowledge/attitude of nurses of Family Health Strategy. Rev Esc Enferm USP. 2019;53:e03482. DOI: http://dx.doi.org/10.1590/S1980-220X2018018103482.

18. Cabral JR, Alencar DL, Vieira JCM, Cabral LR, Ramos VP, Vasconcelos EMR. Oficinas de educação em saúde com idosos: uma estratégia de promoção de qualidade de vida. Rev Enf. 2015 Jul-Dez;1(2):71-75.

19. Tavares MFL, Rocha RM, Bittar CML, Petersen CB, Andrade M. A promoção de saúde no ensino profissional: desafios na Saúde e a necessidade de alcançar outros setores. Ciênc Saúde Coletiva. 2016; 21(6):1799-1808.

20. Pitz AF. Matsuchita HLP. Importância da Educação em Saúde na Terceira Idade. UNICIÊNCIAS. 2015;19(2): 161-168.

21. Guedes MBOG, Lima KC, Caldas CP. Veras RP. Apoio social e o cuidado integral à saúde do idoso. Physis. 2017; 27(4):1185-1204.

22. Srinivasan S et al. Sexuality and the Older Adult. Curr Psychiatry Rep. 2019; 21(97). https://doi.org/10.1007/s11920-019-1090-4

23. Carrasco MH et al. Características de la esfera sexual en pacientes adultos Mayores. Semergen. 2019;45(1):37-43

24. Komlenac N. Siler H. Hochleitner M. Medical students indicate the need for increased Sexuality Education at na Austrian Medical University. Sex Med. 2019:(7):318-325.

\section{Agradecimentos:}

Agradecimento aos discentes e docentes envolvidos na ação e à equipe da UBS.

RECEBIDO: 11/03/2020

ACEITO: 09/06/2020 